\title{
Avaliação do risco para diabetes mellitus tipo 2 em uma clínica no oeste do Paraná
}

\author{
Risk assessment for type 2 diabetes mellitus in a clinic western Paraná \\ Evaluacíon del riesgo de diabetes mellitus tipo 2 em uma clínica del oeste de Paraná
}

Recebido: 14/07/2021 | Revisado: 19/07/2021 | Aceito: 20/07/2021 | Publicado: 28/07/2021

\author{
Kenji Rocha Nakahara \\ ORCID: https://orcid.org/0000-0001-7098-2730 \\ Centro Universitário Fundação Assis Gurgacz. Brasil \\ E-mail: kenjirocha@hotmail.com \\ Marise Vilas Boas Pescador \\ ORCID: https://orcid.org/0000-0003-3718-1063 \\ Centro Universitário Fundação Assis Gurgacz. Brasil \\ E-mail: marisevilasboas@hotmail.com \\ João Vytor Pagnussat \\ ORCID: https://orcid.org/0000-0002-2921-4980 \\ Centro Universitário Fundação Assis Gurgacz. Brasil \\ E-mail: joaopagnussat@gmail.com \\ Julia Damasio Gai \\ ORCID: https://orcid.org/0000-0003-1873-7417 \\ Centro Universitário Fundação Assis Gurgacz. Brasil \\ E-mail: juliadgai@gmail.com
}

\begin{abstract}
Resumo
Diabetes mellitus é um sério problema de saúde pública cuja detecção e tratamento precoce são importantes para a redução das taxas de morbimortalidade. Esse estudo teve como objetivo avaliar a prevalência de indivíduos com possível pré-diabetes e alto risco para diabetes mellitus tipo 2 em pacientes e acompanhantes de uma clínica no oeste do Paraná. Realizou-se a aplicação do questionário adaptado da American Diabetes Association para estimar o escore de risco em 223 participantes. 38,2\% dos indivíduos sem diagnóstico prévio de diabetes apresentaram alto risco para a doença. Os fatores de risco estatisticamente significantes para a doença foram: idade avançada, histórico familiar positivo, hipertensão e índice de massa corporal aumentado. Concluiu-se que grande parcela da amostra possui risco aumentado para diabetes tipo 2 e provável pré-diabetes. A utilização de ferramentas práticas e efetivas como o questionário de risco da American Diabetes Association pode apresentar benefícios para políticas em saúde pública.

Palavras-chave: Diabetes mellitus; Estado pré-diabético; Fatores de risco.
\end{abstract}

\begin{abstract}
Diabetes mellitus is a serious public health problem which the early detection and treatment are extremely important to reduce the morbimortality rates. This study aims to evaluate the prevalence of individuals with possible prediabetes and high risk for type 2 diabetes mellitus in patients and companions at a clinic in western Paraná. A questionnaire adapted from American Diabetes Association was conducted to estimate the risk score in 223 participants. $38.2 \%$ of patients without diagnosis of diabetes presented a high risk for the disease. Statistically significant risk factors were: advanced age, positive family history, hypertension and increased mass index. The study concluded that a large part of the sample is at increased risk for type 2 diabetes and is likely to have prediabetes. The use of practical and effective tools such as the American Diabetes Association risk questionnaire may benefit public health policies.
\end{abstract}

Keywords: Diabetes mellitus; Prediabetic state; Risk factors.

\section{Resumen}

La diabetes mellitus es un grave problema de salud pública cuya detección y tratamiento precoces son importantes para reducir las tasas de morbilidad y mortalidad. Este estudio tuvo como objetivo evaluar la prevalencia de individuos con posible prediabetes y alto riesgo de diabetes mellitus tipo 2 en pacientes y cuidadores de una clínica del oeste de Paraná. Se aplicó un cuestionario adaptado de la American Diabetes Association para estimar la puntuación de riesgo en 223 participantes. El 38,2\% de las personas sin un diagnóstico previo de diabetes tenían un alto riesgo de contraer la enfermedad. Los factores de riesgo estadísticamente significativos para la enfermedad fueron: mayor edad, antecedentes familiares positivos, hipertensión y aumento del índice de masa corporal. Se concluyó que una gran parte de la muestra tiene un mayor riesgo de diabetes tipo 2 y probable prediabetes. El uso de herramientas prácticas y efectivas como el Cuestionario de riesgo de American Diabetes Association puede tener beneficios para la política de salud pública.

Palabras clave: Diabetes mellitus; Estado pré-diabético; Factores de riesgo. 


\section{Introdução}

O Diabetes mellitus (DM) e suas complicações agudas e crônicas são as principais causas de mortalidade e morbidade no mundo (Roth et al. 2018; Murray et al. 2012). De acordo com projeções da International Diabetes Federation (IDF), esperase um aumento exponencial no número de indivíduos acometidos pelo DM nos próximos anos. A prevalência de adultos (2079 anos) com DM e pré-diabetes no ano 2019 foi de 463 milhões e 373,9 milhões, respectivamente (9,3\% e 7,5\% da população mundial nessa faixa etária) (International Diabetes Federation [IDF], 2019). As estimativas apontam que o número de doentes continuará aumentando em razão do envelhecimento e crescimento populacional; urbanização e a alta prevalência do sedentarismo; além do aumento de consumo de alimentos industrializados, o que está diretamente associado a obesidade (Shaw, Sicree \& Zimmet, 2010). Estima-se que até 2045 700,2 milhões de pessoas em idade produtiva apresentarão diabetes, demonstrando um aumento de 51,2\% em menos de três décadas (IDF, 2019)3.

Além do aumento expressivo no número de casos da doença, muitas pessoas não tem conhecimento sobre sua condição clínica. Em 2019, estimou-se que cerca 50,1 \% dos portadores de diabetes com idade entre 20 - 79 anos ignoravam sua condição, ou seja, uma a cada duas pessoas permanece sem diagnóstico e desconhece sua doença (IDF, 2019).

O DM é uma doença crônica onde há um estado de hiperglicemia decorrente de uma série de condições genéticas e ambientais que resultam em disfunção da secreção de insulina e destruição progressiva da massa de células $\beta$ do pâncreas e/ou da resistência dos tecidos ao uso da insulina produzida (Ferrannini et al., 2005; Matveyenko \& Butler, 2008). Uma vez que o estado de hiperglicemia instala-se, o indivíduo está sujeito aos efeitos deletérios dessa condição, sendo que o risco principal é o comprometimento dos vasos resultando no desenvolvimento de complicações microvasculares (retinopatia, nefropatia e neuropatia) e macrovasculares (acidente vascular encefálico, doença arterial obstrutiva periférica, doença arterial coronariana). $\mathrm{O}$ acometimento difuso aos vasos associado às alterações metabólicas do diabetes são os maiores causadores de morbidade e mortalidade da doença (Skyler et al., 2017).

O significativo número de casos de DM não diagnosticados é resultado da combinação de sistemas públicos de saúde com inúmeras fragilidades, da falta de conhecimento da população e dos profissionais da saúde sobre a doença, além da lenta progressão da doença, que fazem com que exista um longo intervalo de tempo até o surgimento dos primeiros sintomas (Beagley, Guariguata, Weil \& Motala, 2014; Maina, Ndegwa, Njenga, \& Muchemi, 2010). A principal implicação da falta de sensibilidade na realização desses diagnósticos é o alto índice de morbidade ao qual esse grupo está sujeito, ocasionando uma enorme busca por serviços de saúde e, consequentemente, maiores gastos com saúde publica (Asmelash \& Asmelash, 2019). Inúmeros indivíduos, ao receberem o diagnóstico dessa patologia, apresentam variadas disfunções e danos teciduais já instalados, visto que apresentam risco aumentado para complicações microvasculares e macrovasculares (Skyler et al., 2017; American Diabetes Association [ADA], 2014).

Diversas pesquisas têm demonstrado o potencial de prevenção e atraso na evolução do DM tipo 2 por meio de medidas de mudança no estilo de vida ou por meio de terapias farmacológicas (Tuomilehto et al., 2001; Knowler et al.,2002; Chiasson et al., 2002) em pacientes que possuem intolerância à glicose, evidenciando que a identificação e abordagem de indivíduos com risco aumentado para o desenvolvimento da doença, ou mesmo o pré-diabetes, é de fundamental importância para minimizar o impacto dessa patologia sobre a qualidade de vida de seus portadores e sobre os gastos com saúde pública (Bang et al., 2009).

A detecção e o tratamento precoce da doença são essenciais para a redução da carga global decorrente das complicações do DM (Bang et al., 2009). Entretanto, promover o rastreamento dessa condição com testes específicos de tolerância à glicose em todos os adultos de populações numerosas se torna economicamente inviável para qualquer sistema de saúde que possua ampla cobertura populacional. Assim, estratégias mais oportunas de rastreio, por meio de ferramentas práticas e efetivas, podem apresentar impacto positivo nos indicadores dessa patologia (Heikes, Eddy, Arondekar \& 
Schlessinger, 2008).

Dessa forma, o questionário de risco de diabetes da American Diabetes Association (ADA) tem sido recomendado como uma opção de rastreamento para orientar os profissionais da saúde sobre a necessidade de realização de testes diagnósticos como medidas de glicemia de jejum, teste oral de tolerância a glicose (TOTG) ou hemoglobina glicada (HbA1c) (Woo et al., 2017). Esse questionário, o qual passou por alterações a partir de um modelo elaborado em 2009 utilizando dados do NHANES 1999-2004, tem como objetivo realizar a triagem por meio de um sistema de pontuações e cuja aplicação pode ser feita em inúmeras situações e ambientes como salas de espera em consultórios médicos ou mesmo pela internet, utilizando um método rápido, simples e eficiente de perguntas e repostas (Heikes et al., 2008; Woo et al., 2017).

O objetivo desse estudo foi, portanto, identificar a prevalência de indivíduos com risco alto para desenvolvimento de DM tipo 2 (DM2) e provavelmente pré-diabéticos e em usuários e acompanhantes de um centro de saúde multidisciplinar na região oeste do Paraná, utilizando como instrumento de pesquisa o questionário da ADA para avaliação de risco de diabetes, comparando os resultados encontrados com os descritos na literatura nacional e internacional.

\section{Metodologia}

Realizou-se um estudo observacional do tipo transversal descritivo quantitativo tendo como suporte metodológico Marconi e Lakatos (2021), entre os meses de março a agosto de 2020 no complexo de Clínicas do Centro Universitário Fundação Assis Gurgacz (FAG), em Cascavel-PR. O projeto de pesquisa foi aprovado no dia 29 de fevereiro de 2020 pelo Comitê de Ética e Pesquisa na Plataforma Brasil, com o número do parecer 3.891.006, expedido pelo Centro Universitário FAG. O Termo de Consentimento Livre e Esclarecido (TCLE) foi assinado por todos os participantes após os procedimentos envolvidos no estudo terem sido detalhadamente explicados.

A população foi constituída por indivíduos que atendiam aos seguintes critérios de inclusão: maiores de 18 anos que buscaram atendimento ambulatorial nas Clínicas do campus da FAG, incluindo acompanhantes, e que haviam aceitado participar do estudo.

A pesquisa utilizou o questionário "Do you have prediabetes?", em tradução livre "Você tem pré-diabetes?", da Sociedade Americana de Diabetes, adaptado a partir do estudo de Bang et al. (2009), que passou por processo de tradução e nova adaptação seguindo modelos brasileiros consolidados (Beaton, Bombardier, Guillemin \& Ferraz, 2000; Coutinho-Myrrha et al, 2014) para a sua validação e utilização.

O questionário apresentava os seguintes quesitos: paciente diabético (sim ou não); sexo (masculino ou feminino); idade (com pontos de corte de 40, 50 e 60 anos); histórico de diabetes gestacional (sim ou não, aplicável para mulheres); histórico familiar de diabetes em mãe, pai, irmã ou irmão; diagnóstico de hipertensão; sedentarismo através da ausência de atividade física e índice de massa corpórea (teste através de uma variável binária de peso e altura). Cada critério apresentava sua pontuação de acordo com a presença dos fatores de risco aplicáveis. O status corpóreo foi realizado por meio do índice de massa corpórea (IMC) onde o entrevistado era selecionado dentre 4 grupos de pontuação: 0 pontos se IMC < 25 (normal ou baixo peso); 1 ponto com IMC entre 25-30 (sobrepeso), 2 pontos com IMC entre 30-40 (obesidade grau I e II) e 3 pontos com IMC $>40$ (obesidade grau III).

As fichas avaliativas (Figura 1) eram disponibilizadas para os participantes em meio impresso. As respostas obtidas foram realizadas através de autopreenchimento de forma individual e anônima. Os entrevistados foram orientados a ler as informações e responder de acordo com entendimento destas. 
Figura 1. Questionário de risco adaptado.

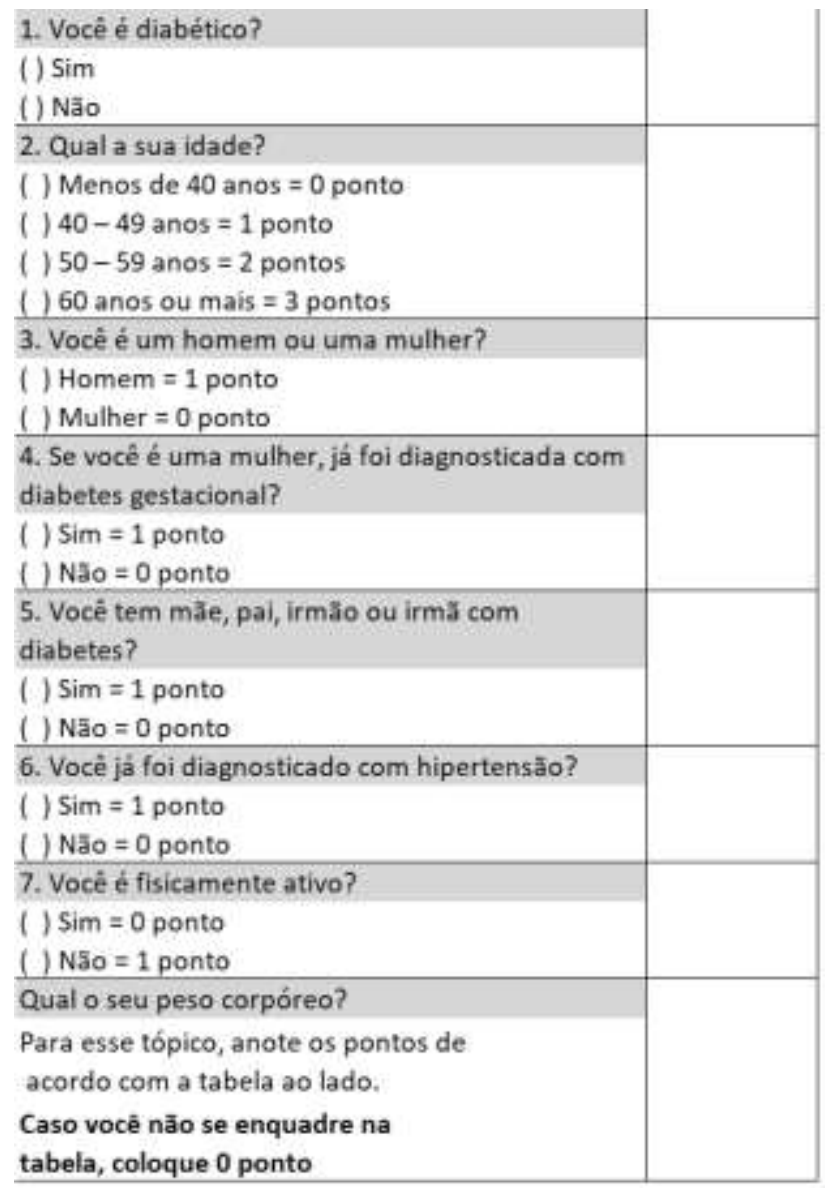

\begin{tabular}{c|c|c|c|}
\hline Altura $(\mathrm{m})$ & \multicolumn{3}{|c|}{ Peso $(\mathrm{kg})$} \\
\hline 1,47 & $54-64$ & $65-86$ & $87+$ \\
\hline 1,5 & $56-67$ & $67-89$ & $90+$ \\
\hline 1,52 & $58-69$ & $69-92$ & $93+$ \\
\hline 1,55 & $60-71$ & $72-95$ & $96+$ \\
\hline 1,57 & $62-74$ & $74-98$ & $99+$ \\
\hline 1,6 & $64-76$ & $77-102$ & $102+$ \\
\hline 1,63 & $66-78$ & $79-105$ & $105+$ \\
\hline 1,65 & $68-81$ & $82-108$ & $109+$ \\
\hline 1,68 & $70-84$ & $84-112$ & $112+$ \\
\hline 1,7 & $72-86$ & $87-115$ & $116+$ \\
\hline 1,73 & $74-89$ & $89-118$ & $119+$ \\
\hline 1,75 & $77-92$ & $92-122$ & $122+$ \\
\hline 1,78 & $79-94$ & $95-126$ & $126+$ \\
\hline 1,8 & $81-97$ & $98-129$ & $130+$ \\
\hline 1,83 & $83-100$ & $100-133$ & $133+$ \\
\hline 1,85 & $86-103$ & $103-137$ & $137+$ \\
\hline 1,88 & $88-105$ & $106-141$ & $141+$ \\
\hline 1,91 & $91-108$ & $109-144$ & $144+$ \\
\hline 1,93 & $93-111$ & $112-148$ & $149+$ \\
\cline { 2 - 4 } & 1 & 2 & 3 \\
\cline { 2 - 4 } & ponto & pontos & pontos \\
\cline { 2 - 4 } & & &
\end{tabular}

Fonte: Autores (2020).

Validou-se apenas os questionários obrigatoriamente respondidos na presença dos pesquisadores e em perfeito estado (sem danos, desenhos ou rasuras). Os materiais rasurados ou incompletos foram desconsiderados para tabulação dos dados. Após o preenchimento foi calculado o risco de cada indivíduo atribuindo o escore total.

Com base nas instruções do questionário aplicado em sua versão final, considerou-se indivíduo provavelmente prédiabético e com risco elevado de desenvolvimento de DM2 as somatórias de pontos que atingiram escore maior ou igual a cinco, conforme estabelecido pela ADA (Beaton et al., 2000).

Para todas as respostas coletadas foram utilizadas estatísticas descritivas tais como frequência absoluta e relativa (porcentagem), e representações gráficas; a fim de caracterizar à amostra e avaliar possíveis relações significativas entre os grupos da situação de diabetes com relação as demais variáveis. Assim, foram realizados testes (qui-quadrado- ) ou Testes exato de Fisher, para o caso de resultados com menos de 5 pacientes.

Todas as estatísticas foram desenvolvidas no software R (R Core Team, 2020) e o nível de significância assumido foi de $5 \%(\mathrm{p}<0,05)$, podendo-se afirmar a existência de associação significativa entre as variáveis qualitativas.

\section{Resultados}

A amostra estudada contemplou 240 participantes. Desse total, 17 questionários foram excluídos por apresentarem danos ou rasuras restando 223 questionários em perfeitas condições. Durante a análise dos formulários 24 indivíduos autodeclararam-se diabéticos e seus dados foram utilizados como forma de comparativo com o grupo de pacientes não diabéticos. 
O total de pessoas incluídas no estudo foram divididas em 3 grupos: grupo com pontuação menor que 5, ou seja, escore negativo para risco de pré-diabetes ou DM 2 (G1); grupo com escore maior ou igual a 5 e autodeclarados não diabéticos, porém considerados pelo resultado do teste, provavelmente pré-diabéticos e com risco elevado para o desenvolvimento de DM 2 (G2) e o último grupo com indivíduos autodeclarados diabéticos (G3).

O G1 foi composto por 123 (55,15\%) indivíduos, enquanto 76 questionários enquadraram-se no grupo G2 (34,08\%) e 24 no grupo G3 (10,77\%).

A análise de cada uma das varáveis aplicadas no questionário utilizado está sintetizada na Tabela 1.

Tabela 1. Caracterização da amostra segundo os grupos: diabéticos, baixo e alto risco para o desenvolvimento da doença.

\begin{tabular}{|c|c|c|c|c|}
\hline Aspectos & $\begin{array}{c}\text { Baixo risco } \\
\text { (G1) }\end{array}$ & $\begin{array}{l}\text { Alto risco } \\
\text { (G2) }\end{array}$ & $\begin{array}{c}\text { Diabéticos } \\
\text { (G3) }\end{array}$ & p-valor \\
\hline \multicolumn{5}{|l|}{ Idade } \\
\hline$<40$ & $88,00 \%(66)$ & $10,67 \%(8)$ & $1,33 \%(1)$ & $<0,001 *$ \\
\hline $40-49$ & $68,42 \%(39)$ & $26,32 \%(15)$ & $5,26 \%(3)$ & \\
\hline $50-59$ & $33,33 \%(16)$ & $50,00 \%(24)$ & $16,67 \%(8)$ & \\
\hline$\geq 60$ & $4,65 \%(2)$ & $67,44 \%(29)$ & $27,91 \%(12)$ & \\
\hline \multicolumn{5}{|l|}{ Sexo } \\
\hline Feminino & $57,72 \%(86)$ & $30,20 \%(45)$ & $12,08 \%(18)$ & 0,198 \\
\hline Masculino & $50,00 \%(37)$ & $41,89 \%(31)$ & $8,11 \%(6)$ & \\
\hline \multicolumn{5}{|c|}{ Diabetes Gestacional (Sexo: Feminino) } \\
\hline Sim & $20,00 \%$ & $40,00 \%(2)$ & $40,00 \%(2)$ & 0,068 \\
\hline Não & $59,03 \%(85)$ & $29,86 \%(43)$ & $11,11 \%(16)$ & \\
\hline \multicolumn{5}{|l|}{ Família com DM } \\
\hline Não & $74,05 \%(97)$ & $22,14 \%(29)$ & $3,82 \%(5)$ & $<0,001 *$ \\
\hline Sim & $28,26 \%(26)$ & $51,09 \%(47)$ & $20,65 \%(19)$ & \\
\hline \multicolumn{5}{|l|}{ Hipertensão } \\
\hline Sim & $13,89 \%(10)$ & $56,94 \%(41)$ & $29,17 \%(21)$ & $<0,001 *$ \\
\hline Não & $74,83 \%$ (113) & $23,18 \%(35)$ & $1,99 \%(3)$ & \\
\hline \multicolumn{5}{|l|}{ Ativo fisicamente } \\
\hline Sim & $64,37 \%(67)$ & $26,44 \%(23)$ & $9,20 \%(8)$ & 0,083 \\
\hline Não & $49,26 \%(56)$ & $38,97 \%(53)$ & $11,76 \%(16)$ & \\
\hline \multicolumn{5}{|l|}{ IMC } \\
\hline$<25$ & $84,85 \%(56)$ & $13,64 \%(9)$ & $1,52 \%(1)$ & $<0,001 *$ \\
\hline $25-30$ & $59,74 \%(46)$ & $27,27 \%(21)$ & $12,99 \%(10)$ & \\
\hline $30-40$ & $31,03 \%(18)$ & $53,45 \%(31)$ & $15,52 \%(9)$ & \\
\hline$\geq 40$ & $13,64 \%(3)$ & $68,18 \%(15)$ & $18,18 \%(4)$ & \\
\hline Escore médio \pm DP & $2,49 \pm 1,22$ & $5,92 \pm 1,17$ & $6,62 \pm 1,17$ & --- \\
\hline Total de pacientes & $55,15 \%(123)$ & $34,08 \%(76)$ & $10,76 \%(24)$ & \\
\hline
\end{tabular}

Legenda: \% - percentagem; IMC - índice de massa corpórea; p-valor - nível de significância; DM - diabetes mellitus; DP - desvio padrão.

Fonte: Autores (2021).

Os fatores de risco idade avançada, histórico familiar positivo para diabetes, hipertensão e índice de massa corporal (IMC) aumentado apresentaram valores estatisticamente significativo para aumento de risco de diabetes e/ou pré-diabetes.

A Figura 2 demonstra a distribuição do resultado de escore do questionário aplicado no estudo para cada grupo; a média encontrada no G1 foi 2,49 $\pm 1,22$; no grupo G2 5,92 $\pm 1,17$ e no grupo G3 6,62 $\pm 1,17$; observou-se que a média do grupo 2 foi semelhante a do grupo com diabetes (G3), e no grupo G1 foi menor que no grupo 3, dado esse que reforça a validade do teste como teste de triagem para investigação de risco de diabetes na população geral. 
Figura 2. Gráfico referente ao escore dos pacientes de acordo com a condição de saúde relativo ao diabetes.

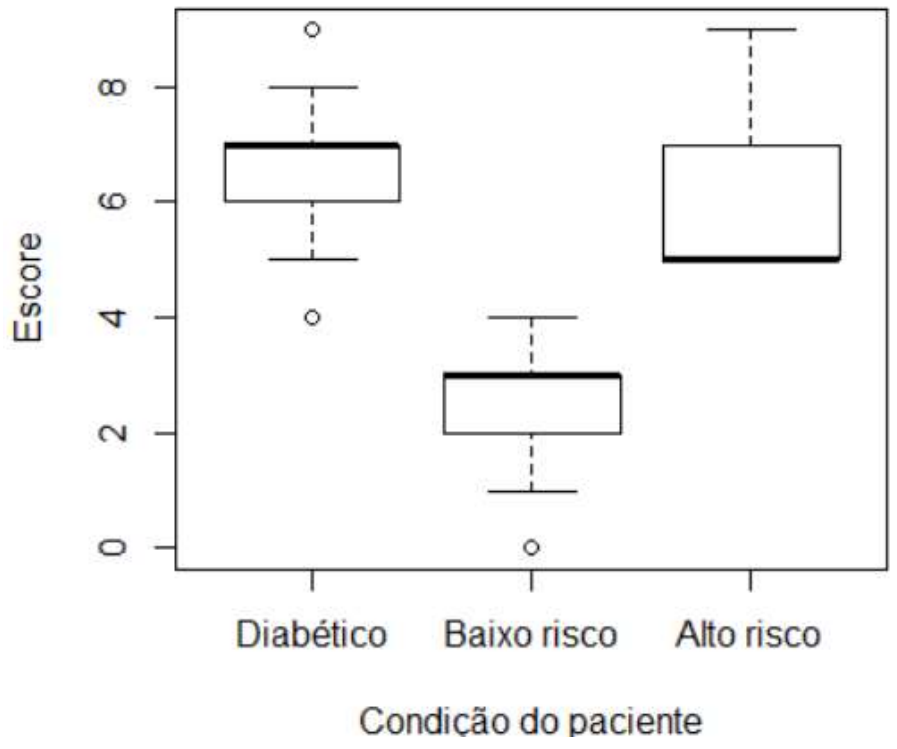

Fonte: Autores (2021).

\section{Discussão}

O objetivo do estudo foi identificar indivíduos provavelmente pré-diabéticos e com risco para desenvolvimento de DM2 em usuários e acompanhantes de um centro de saúde multidisciplinar no oeste do Paraná.

A maioria dos entrevistados do estudo eram do sexo feminino o que corrobora com dados encontrados sobre o predomínio de busca de serviços de saúde pelas mulheres em estudos nacionais (Tomasi et al., 2011). Apesar disso, proporcionalmente o número de homens com alto risco para diabetes encontrado foi maior (41,89\% do total de homem $\mathrm{X}$ $30,20 \%$ do total das mulheres pertenciam ao G2, porém (sem significância estatística), o que pode sugerir um menor cuidado com a condição de saúde pelo sexo masculino, tornando essa população mais vulnerável às taxas de morbimortalidade da doença. Além disso, inúmeros estudos demonstraram a relação do sexo masculino como fator de risco para diabetes. (Bittencourt \& Vinholes, 2013; Schmid, Vollenweider, Waeber \& Marques-Vidal, 2011).

A avaliação do IMC nos pacientes sem diagnóstico de DM2 mostrou que a maioria dos participantes do G1 possuía IMC < 25, visto que $84,85 \%$ dos participantes com pontuação 0 para o critério IMC pertenciam ao grupo de baixo risco. Já no grupo de alto risco a frequência de pontuações observada foi de $9(13,64 \%)$ com IMC < 25; 21 (27,27\%) com IMC 25-29,9; 31 $(53,45 \%)$ com IMC 30-39,9; e $15(68,18 \%)$ com IMC $\geq 40$ demonstrando que a maior parte dos participantes com algum grau de obesidade pertenciam ao G2.

Assim, a importância da obesidade e sobrepeso como fator de risco também foi encontrada neste estudo. Na amostra, a medidas de IMC encontradas foram maiores na população com alto risco se comparada aquela com baixo risco, com resultado estatisticamente significativo. Sendo que $88,16 \%$ da população de alto risco apresentavam IMC $\geq 25$, ou seja apenas $11,84 \%$ desse grupo apresentava peso adequado. Resultado próximo ao estudo conduzido na atenção primária em Cuba que encontrou dados de $80 \%$ da população com DM2 apresentando sobrepeso e obesidade (Naranjo, Rodríguez, Llera \& Aroche 2013). Esse achado é frequente em estudos (Zardo, Bassan, Farias, Diefenthaeler \& Grazziotin, 2015; Bachur, de Morais, Vanoni, Ribeiro \& da Silva Candido, 2018) demonstrando que quanto maior o IMC maior o risco para o desenvolvimento de diabetes.

Em múltiplas analises o aumento da idade tem relação direta com a prevalência de diabetes na população, sendo que no relatório da IDF $9^{\circ}$ edição, 2019 a prevalência estimada da doença na América do Sul e América Central apresenta valores 
progressivamente maiores com a idade (IDF, 2019). A maioria dos participantes da pesquisa estava no grupo etário inferior a de 40 anos (37,19\%). Entretanto no grupo com escore positivo (G2), a maioria dos participantes apresentava idade entre 50 e 59 anos $(31,58 \%)$ e mais de 60 anos $(38,16 \%)$, sendo esse último o maior grupo, indicando a relação proporcional de risco e idade ( $\mathrm{p}<0,001$ ). Uma pesquisa realizada na Turquia também corroborou com esse resultado, onde o aumento de 1 desvio padrão na idade estava associado a um aumento de 1,6 e 1,67 na prevalência de diabetes em mulheres e homens, respectivamente (Satman et al., 2013).

No presente estudo, a incidência de diabetes gestacional foi pequena entre os grupos, não apresentando significância estatística.

Quando pesquisado a presença de casos de diabetes entre familiares de primeiro grau (pai, mãe e irmãos), 92 (36,68\%) questionários apresentaram esse fator de risco positivo. A proporção de respostas no grupo G2 foi de 47 (61,84\%) "Sim" e 29 (38,16\%) "Não" para o histórico familiar positivo. Esse dado corrobora com o estudo realizado na população britânica o qual evidenciou que o histórico familiar positivo para DM em parentes de primeiro grau aumenta o risco em 2,3 vezes em mulheres e 2,7 vezes em homens (Hippisley-Cox, Coupland, Robson, Sheikh, \& Brindle, 2009). Assim, indivíduos com fator genético positivo para diabetes, devem focar esforços em alterar os fatores modificáveis relacionados a estilo de vida, já que apresentam risco maior para o desenvolvimento dessa patologia.

Em relação ao diagnóstico de hipertensão, 51 (25,61\%) indivíduos do estudo apresentavam essa condição. No grupo G2 o número de pessoas autodeclaradas hipertensas foi significativamente maior $(53,94 \%)$ ao passo que a população de baixo risco apresentava baixa positividade para esse fator de risco ( $8,13 \%$ do grupo $\mathrm{G} 1)$. O estudo CARMELA, realizado em centros urbanos da América Latina, também encontrou essa relação identificando a hipertensão como um dos fatores de risco cardiovascular mais comumente associado ao DM2 (hipertensos eram 1,5-2,2 vezes mais propensos a ter diabetes que participantes normotensos) (Escobedo et al., 2009).

Referente à prática de exercícios físicos, 56,05\% (125) participantes consideraram-se sedentários. Quando avaliado isoladamente o grupo de alto risco (G2), $23(30,26 \%)$ integrantes autodeclararam-se fisicamente ativos, ao passo que 53 $(69,74 \%)$ eram sedentários, valores percentuais superiores ao grupo de baixo risco, porém sem significado estatístico. Esse resultado se assemelha ao encontrado em estudos nacionais (Bittencourt \& Vinholes, 2013) e internacionais (Leahy et al., 2015; Islam, Chakrabarti, Islam, Wahab \& Lamoureux, 2016). Uma pesquisa realizada em Bangladesh encontrou uma relação de risco relacionada ao sedentarismo de 1,77 vezes maior para o desenvolvimento de DM (Islam et al., 2016).

Comparando o escore médio obtido no grupo G1 e G2 com o grupo G3, observou-se que o escore médio desse grupo foi semelhante com o grupo G2 e estatisticamente maior que do grupo 1, não foi encontrado comparação semelhante na literatura, porém esse resultado reforça a utilidade do teste como método de triagem populacional para risco de desenvolvimento de diabetes, devendo ser orientado que os indivíduos que obtiveram escore $\geq 5$ realizem testes laboratoriais específicos para diagnóstico de diabetes.

Os limites dos resultados desse estudo foram o desenho transversal que não permitem o estabelecimento de relações de causa e efeito. Além disso, o acesso aos usuários homens que por serem pouco frequentadores do serviço de saúde limitou uma análise estratificada por sexo.

\section{Conclusão}

Os dados obtidos no presente estudo caracterizaram uma população predominantemente feminina, na faixa etária de até 40 anos, com histórico familiar negativo para DM, não hipertensa, sedentária e com sobrepeso ou obesidade. A partir da análise da razão de prevalência, aplicada a todas as variáveis clínicas investigadas, os fatores de risco idade avançada; histórico familiar positivo para diabetes; presença de hipertensão arterial e IMC aumentado demonstraram-se estatisticamente 
significantes para o risco de desenvolvimento de diabetes. O comparativo entre a média do escore dos grupos de alto risco e de baixo risco com o grupo já com diagnóstico de diabetes, reforça a utilidade do questionário aplicado como um teste de triagem populacional. Assim, diante do conhecimento sobre o perfil da população, cabe aos profissionais de saúde identificar fatores de risco com ênfase na promoção de hábitos de vida saudáveis. Reconhecer fatores de risco é fundamental para estabelecer medidas preventivas que impactem no processo saúde doença e na prevenção dessa patologia.

A alta prevalência de diabetes e pré-diabetes representa um grande problema de saúde pública, exigindo ações significativas tanto na prevenção como no diagnóstico da doença. O tratamento precoce previne e modifica a progressão do diabetes, reduzindo suas complicações macro e microvasculares.

Desse modo, questionários de risco de diabetes simples e práticos, tais como o da ADA, poderiam ser amplamente utilizados como opção de rastreamento para orientar a realização de testes laboratoriais diagnósticos específicos na população com alto risco para DM2 a fim de realizar o diagnóstico precoce dessa doença.

Espera-se que os resultados demonstrados por esse estudo permitam expandir o conhecimento sobre os métodos de screening do diabetes, justificando a necessidade de novas pesquisas que realizem o seguimento de pacientes com fatores de riscos para a doença, especialmente os de alto risco, visando o seu diagnostico precoce para que se possa comprovar a utilidade do questionário da ADA no âmbito da saúde pública e na modificação da história natural da doença.

\section{Referências}

American Diabetes Association. (2014). Diagnosis and classification of diabetes mellitus. Diabetes care, 37(Supplement 1), S81-S90.

Asmelash, D., \& Asmelash, Y. (2019). The burden of undiagnosed diabetes mellitus in adult African population: a systematic review and metaanalysis. Journal of diabetes research, 2019.

Bachur, C. K., de Morais, A. F. P., Vanoni, H. D. C., Ribeiro, I. S., \& da Silva Candido, S. (2018). Stratification of risk factors for type 2 diabetes mellitus in health professionals/Estratificacao dos fatores de risco de diabetes mellitus tipo 2 em profissionais da saude. Demetra: Food, Nutrition \& Health, 13(4), 965975 .

Bang, H., Edwards, A. M., Bomback, A. S., Ballantyne, C. M., Brillon, D., Callahan, M. A., \& Kern, L. M. (2009). Development and validation of a patient self-assessment score for diabetes risk. Annals of internal medicine, 151(11), 775-783.

Beagley, J., Guariguata, L., Weil, C., \& Motala, A. A. (2014). Global estimates of undiagnosed diabetes in adults. Diabetes research and clinical practice, 103(2), 150-160.

Beaton, D. E., Bombardier, C., Guillemin, F., \& Ferraz, M. B. (2000). Guidelines for the process of cross-cultural adaptation of self-report measures. Spine, 25(24), 3186-3191.

Bittencourt, A., \& Vinholes, D. B. (2013). Estimativa do risco para diabetes mellitus tipo 2 em bancários da cidade de Tubarão, estado de Santa Catarina, Brasil. Scientia Medica, Porto Alegre, 23(2), 82-89.

Chiasson, J. L., Josse, R. G., Gomis, R., Hanefeld, M., Karasik, A., \& Laakso, M. (2002). Acarbose for prevention of type 2 diabetes mellitus: the STOPNIDDM randomised trial. The Lancet, 359(9323), 2072-2077.

Coutinho-Myrrha, M. A., Dias, R. C., Fernandes, A. A., Araújo, C. G., Hlatky, M. A., Pereira, D. G., \& Britto, R. R. (2014). Duke Activity Status Index for cardiovascular diseases: validation of the Portuguese translation. Arquivos brasileiros de cardiologia, 102, 383-390.

Escobedo, J., Buitrón, L. V., Velasco, M. F., Ramírez, J. C., Hernández, R., Macchia, A., Pellegrini, F., Schargrodsky, H., Boissonnet, C., Champagne, B. M. (2009). High prevalence of diabetes and impaired fasting glucose in urban Latin America: the CARMELA Study. Diabetic Medicine, 26(9), 864-871.

Ferrannini, E., Gastaldelli, A., Miyazaki, Y., Matsuda, M., Mari, A., \& DeFronzo, R. A. (2005). $\beta$-Cell function in subjects spanning the range from normal glucose tolerance to overt diabetes: a new analysis. The Journal of Clinical Endocrinology \& Metabolism, 90(1), 493-500.

Heikes, K. E., Eddy, D. M., Arondekar, B., \& Schlessinger, L. (2008). Diabetes Risk Calculator: a simple tool for detecting undiagnosed diabetes and prediabetes. Diabetes care, 31(5), 1040-1045.

Hippisley-Cox, J., Coupland, C., Robson, J., Sheikh, A., \& Brindle, P. (2009). Predicting risk of type 2 diabetes in England and Wales: prospective derivation and validation of QDScore. Bmj, 338 .

International Diabetes Federation. (2019). IDF Diabetes Atlas, 9th edn. Brussels, Belgium: International Diabetes Federation.

Islam, F. M., Chakrabarti, R., Islam, M. T., Wahab, M., \& Lamoureux, E. (2016). Prediabetes, diagnosed and undiagnosed diabetes, their risk factors and association with knowledge of diabetes in rural Bangladesh: The Bangladesh Population-based Diabetes and Eye Study. Journal Diabetes 8: $260-8$. 
Research, Society and Development, v. 10, n. 9, e36410918249, 2021

(CC BY 4.0) | ISSN 2525-3409 | DOI: http://dx.doi.org/10.33448/rsd-v10i9.18249

Knowler, W. C., Barrett-Connor, E., Fowler, S. E., Hamman, R. F., Lachin, J. M., Walker, E. A., \& Nathan, D. M. (2002). Reduction in the incidence of type 2 diabetes with lifestyle intervention or metformin. New England journal of medicine, 346(6), 393-403.

Leahy, S., O'Halloran, A. M., O'Leary, N., Healy, M., McCormack, M., Kenny, R. A., \& O'Connell, J. (2015). Prevalence and correlates of diagnosed and undiagnosed type 2 diabetes mellitus and pre-diabetes in older adults: Findings from the Irish Longitudinal Study on Ageing (TILDA). Diabetes research and clinical practice, $110(3), 241-249$.

Maina, W. K., Ndegwa, Z. M., Njenga, E. W., \& Muchemi, E. W. (2010). Knowledge, attitude and practices related to diabetes among community members in four provinces in Kenya: a cross-sectional study. Pan African Medical Journal, 7(1).

Marconi, M. A., \& Lakatos, E. M. (2021). Técnicas de pesquisa. (9a ed.). Atlas.

Matveyenko, A. V., \& Butler, P. C. (2008). Relationship between $\beta$-cell mass and diabetes onset. Diabetes, Obesity and Metabolism, 10, 23 -31.

Murray, C. J., Vos, T., Lozano, R., Naghavi, M., Flaxman, A. D., Michaud, C., \& Haring, D. (2012). Disability-adjusted life years (DALYs) for 291 diseases and injuries in 21 regions, 1990-2010: a systematic analysis for the Global Burden of Disease Study 2010. The lancet, 380(9859), 2197-2223.

Naranjo, A. A., Rodríguez, Á. Y., Llera, R. E., \& Aroche, R. (2013). Diabetes risk in a Cuban primary care setting in persons with no known glucose abnormalities. MEDICC review, 15, 16-19.

Roth, G. A., Abate, D., Abate, K. H., Abay, S. M., Abbafati, C., Abbasi, N., \& Borschmann, R. (2018). Global, regional, and national age-sex-specific mortality for 282 causes of death in 195 countries and territories, 1980-2017: a systematic analysis for the Global Burden of Disease Study 2017. The Lancet, 392(10159), 1736-1788.

Satman, I., Omer, B., Tutuncu, Y., Kalaca, S., Gedik, S., Dinccag, N., \& Tuomilehto, J. (2013). Twelve-year trends in the prevalence and risk factors of diabetes and prediabetes in Turkish adults. European journal of epidemiology, 28(2), 169-180.

Schmid, R., Vollenweider, P., Waeber, G., \& Marques-Vidal, P. (2011). Estimating the risk of developing type 2 diabetes: a comparison of several risk scores: the Cohorte Lausannoise study. Diabetes Care, 34(8), 1863-1868.

Shaw, J. E., Sicree, R. A., \& Zimmet, P. Z. (2010). Global estimates of the prevalence of diabetes for 2010 and 2030. Diabetes research and clinical practice, $87(1), 4-14$.

Skyler, J. S., Bakris, G. L., Bonifacio, E., Darsow, T., Eckel, R. H., Groop, L., \& Ratner, R. E. (2017). Differentiation of diabetes by pathophysiology, natural history, and prognosis. Diabetes, 66(2), 241-255.

Tomasi, E., Facchini, L. A., Thumé, E., Piccini, R. X., Osorio, A., da Silveira, D. S., \& Maia, M. D. S. (2011). Characteristics of primary healthcare service use in the southern and northeastern regions of Brazil: differences by care model. Ciencia \& saude coletiva, 16(11), 4395.

Tuomilehto, J., Lindström, J., Eriksson, J. G., Valle, T. T., Hämäläinen, H., Ilanne-Parikka, P., \& Uusitupa, M. (2001). Prevention of type 2 diabetes mellitus by changes in lifestyle among subjects with impaired glucose tolerance. New England Journal of Medicine, 344(18), 1343-1350.

Woo, Y. C., Lee, C. H., Fong, C. H., Tso, A. W., Cheung, B. M., \& Lam, K. S. (2017). Validation of the diabetes screening tools proposed by the American Diabetes Association in an aging Chinese population. PLoS One, 12(9), e0184840.

Zardo, M., Bassan, M. B., Farias, K., Diefenthaeler, H., \& Grazziotin, N. A. (2015). Rastreamento de fatores de risco para diabetes tipo 2 em trabalhadores de uma indústria da cidade de Concórdia-SC. Perspectiva, Erechim, 39(145), 85-95. 